\title{
A Surgical Case of Heterotopic Thymic Carcinoma Coexisting With Hepatocellular Carcinoma
}

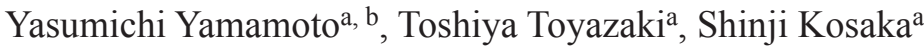

\begin{abstract}
Although thymomas arising in ectopic regions other than the upper anterior mediastinum are well known, thymic carcinomas in ectopic locations are very rare. On the other hand, the therapeutic strategies for synchronous double primary cancers arising from different organs are still controversial. We have experienced a case of thymic carcinoma in an ectopic location with a coexisting hepatocellular carcinoma (HCC) for which management presented a number of difficulties. A 67-year-old man with HCC presented with a slow-growing nodular shadow in the left lower anterior mediastinum, i.e. the left cardiophrenic angle. During 7 years of intensive treatment for the HCC, the mediastinal nodule, which had been suspected clinically to be a lymph node metastasis from the HCC, grew to $4 \mathrm{~cm}$ in diameter. Surgical intervention for the mediastinal nodule was carried out for treatment and definitive diagnosis using a mediastinoscope inserted via a transverse xyphoid incision. Intraoperative pathological diagnosis from frozen sections of the mediastinal nodule revealed thymic carcinoma, and therefore the procedure was converted to thymothymectomy via a median sternotomy. Despite postoperative multimodality treatment, the patient died due to multifocal metastasis and pleuritis carcinomatosa from the thymic carcinoma within 7 months after the thymothymectomy. The unusual location of the thymic carcinoma in this case and the synchronous nature of the coexisting primary HCC resulted in delay of surgical resection for the former. More intensive observation and early intervention in the form of diagnostic or therapeutic resection should be performed for any mediastinal nodule that appears during the course of treatment for HCC.
\end{abstract}

Keywords: Thymic carcinoma; Ectopic location; Mediastinoscopy; Synchronous double primary cancer; Therapeutic management

Manuscript accepted for publication August 13, 2015

aDepartment of Thoracic Surgery, Shimane Prefectural Central Hospital, Shimane, Japan

${ }^{\mathrm{b}}$ Corresponding Author: Yasumichi Yamamoto, Shimane Prefectural Central Hospital, 4-1-1 Himebara, Izumo, Shimane 693-8555, Japan.

Email: yyama@spch.izumo.shimane.jp

doi: http://dx.doi.org/10.14740/jmc2267w

\section{Introduction}

It is well known that thymomas may arise from ectopic thymic tissue in the head and neck region [1]. By contrast, reports of ectopic thymic carcinoma are very limited in view of its rarity, except for carcinoma showing thymus-like elements of the thyroid, so called CASTLE, whose pathogenesis is suspected to be a remnant of the branchial pouch from which the thyroid gland originates, and is thus not ectopic thymus tissue [2-8].

On the other hand, therapeutic strategies for synchronous double primary cancers arising from different organs, such as thymic carcinoma and hepatocellular carcinoma ( $\mathrm{HCC}$ ), are still controversial. In general, the more aggressive, advanced lesion with the poorest prognosis is given initial treatment priority. Most authors agree that treatment of HCC should take priority over any extra-hepatic primary malignant neoplasm $[9,10]$.

\section{Case Report}

A 67-year-old man presented because of a slow-growing nodular shadow in the left lower anterior mediastinum that had been revealed by chest computed tomography (CT), without any subjective symptoms. Physical examination revealed no abnormality other than a surgical scar. Chest X-ray examination showed no abnormalities. Laboratory data were compatible with liver cirrhosis. Liver cirrhosis had developed due to hepatitis virus infection following blood transfusion when being treated for a serious injury, and HCC had been subsequently confirmed by needle aspiration biopsy. The HCC had been treated by percutaneous ethanol injection in 2002, transarterial embolization followed by radiofrequency ablation in 2006, and transarterial injection in 2007. Finally, the residual HCC had been resected in December 2007. The definitive pathological diagnosis was moderately differentiated HCC.

Retrospective examination of the chest CT scan taken in May 2002, just before initial treatment of the HCC, revealed a small nodule in the lower anterior mediastinum at the left cardiophrenic angle (Fig. 1a). This slow-growing mediastinal nodule had been initially detected by radiology in June 2006, but the clinical decision made by the attending gastroenterologist was that treatment of the HCC should be prioritized over pathological diagnosis of the nodule (Fig. 1b). The tentative preoperative diagnosis of the mediastinal nodule was metastat- 

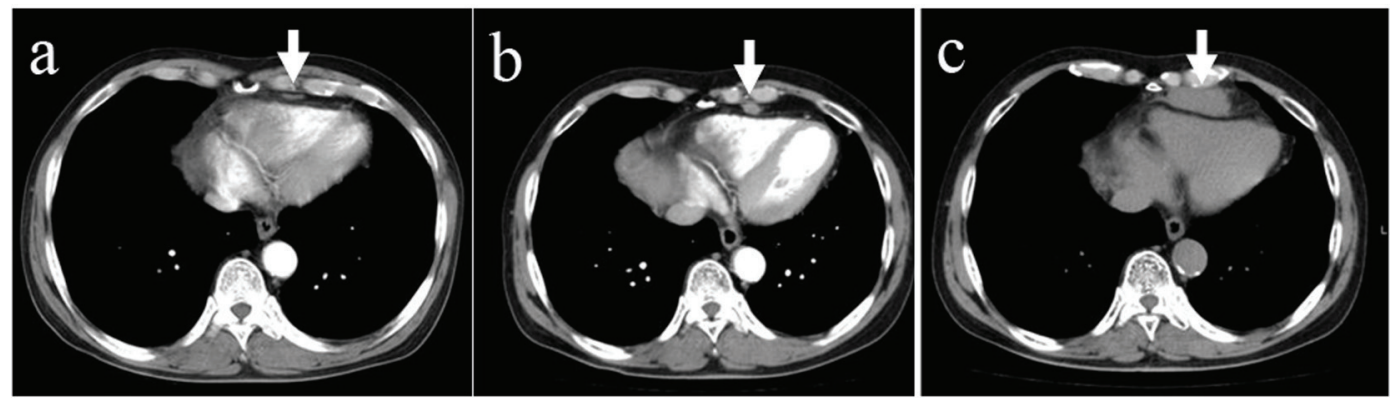

Figure 1. CT findings over a 7-year period. A small nodule was observed in May 2002 (a), and by June 2006 this had grown to an elliptical shape (b), finally becoming a $4 \times 3 \mathrm{~cm}$ tumor compressing the right ventricle by February 2009 (c). Arrow: tumor.

ic lymphadenopathy from HCC. By February 2009, the tumor had grown to $4 \mathrm{~cm}$ in diameter (Fig. 1c). The patient consulted our department and we elected to perform en bloc lymphadenectomy using video-associated mediastinoscopy for pathological confirmation and curative resection of the lymph node metastasis. Preoperative chest and abdominal CT, brain magnetic resonance imaging, and bone scintigraphy confirmed absence of metastasis at any other sites.

\section{Operative procedures}

In February 2009, a mediastinoscope was inserted into the retrosternum via a $5-\mathrm{cm}$ transverse skin incision at the xyphoid. The tumor was found to adhere to the pericardium, left parietal pleura and diaphragm, but not to the left lung. We dissected the tissue between the tumor and the pericardium, and resected part of the left partial pleura and the superficial muscle layer of the diaphragm. Macroscopically, the tumor was removed completely. Rapid pathological diagnosis during the operation revealed poorly differentiated adenocarcinoma, which was quite different from the HCC primary. The tumor had no relationship with the lung parenchyma, and therefore we diagnosed it as adenocarcinoma of the thymus, and converted the operative procedure to thymothymectomy via a median sternotomy. Thymectomy with resection of about $5 \mathrm{~cm}^{2}$ of the pericardium and a one-third area of the full thickness of the left diaphragm was performed to ensure at least a $1-\mathrm{cm}$ free margin from the outer border of the adherent tumor. The resulting defect in the diaphragm was repaired by direct suturing, and the pericardial defect was reconstructed using a polytetrafluoethylene (Gore $\left.\operatorname{Tex}^{\circledR}\right)$ patch membrane. Upper mediastinal lymph node dissection was not performed. The total operation time was $250 \mathrm{~min}$, and the bleeding volume was $150 \mathrm{~mL}$.

\section{Pathological findings}

The tumor was a multi-lobulated mass measuring $65 \times 60 \times 20$ $\mathrm{mm}$ and its cut surface was white-grayish. Microscopically, it consisted of undifferentiated (Fig. 2a, b) squamous cells (Fig. $3 \mathrm{a}, \mathrm{b}$ ) and sarcomatoid carcinoma (Fig. 4a, b). By area, around $60 \%$ of the tumor was undifferentiated, $20 \%$ was squamous cells, and $20 \%$ was sarcomatoid carcinoma. Although invasion into the surface of the excised pericardium and pericardial fat was evident microscopically, the excisional margin was free of any tumor cells. Immunohistochemistry showed that the tumor was positive for CKAE1/AE3, CK7, vimentin, bcl-2 and CD5, weakly positive for chromogranin A, and negative for AFP, HMB45, S-100, CK20 and c-KIT. The associated lymphocytes were mature, showing scant positivity for CD30 and negativity for ALK. The definitive diagnosis was T3N0M0 thymic carcinoma, pathological stage III, based on the WHO criteria [1].

\section{Postoperative treatment}

Postoperative irradiation targeting the excisional margin was
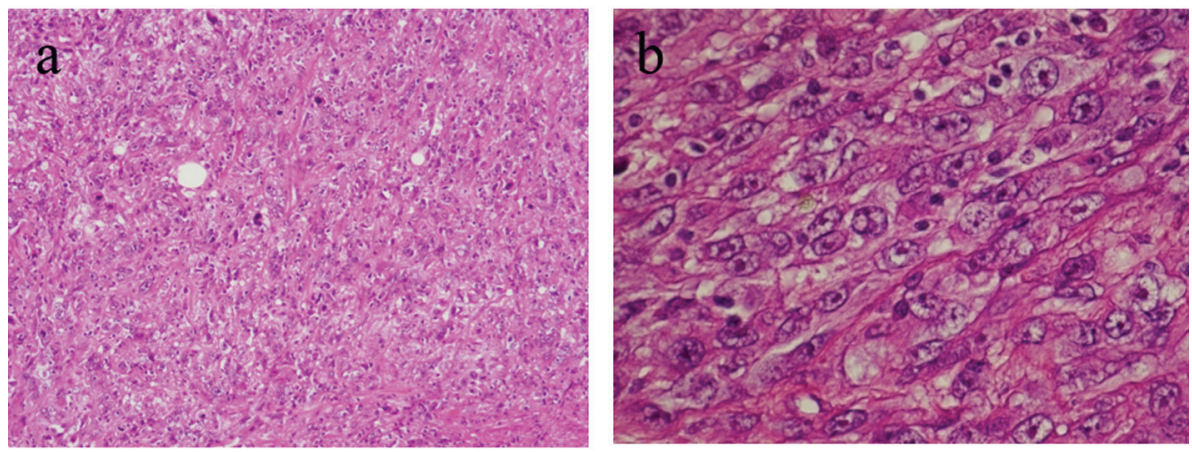

Figure 2. The undifferentiated carcinoma showed sheet-like proliferation without any particular structure or differentiation (a), consisting of small round cells (b). 


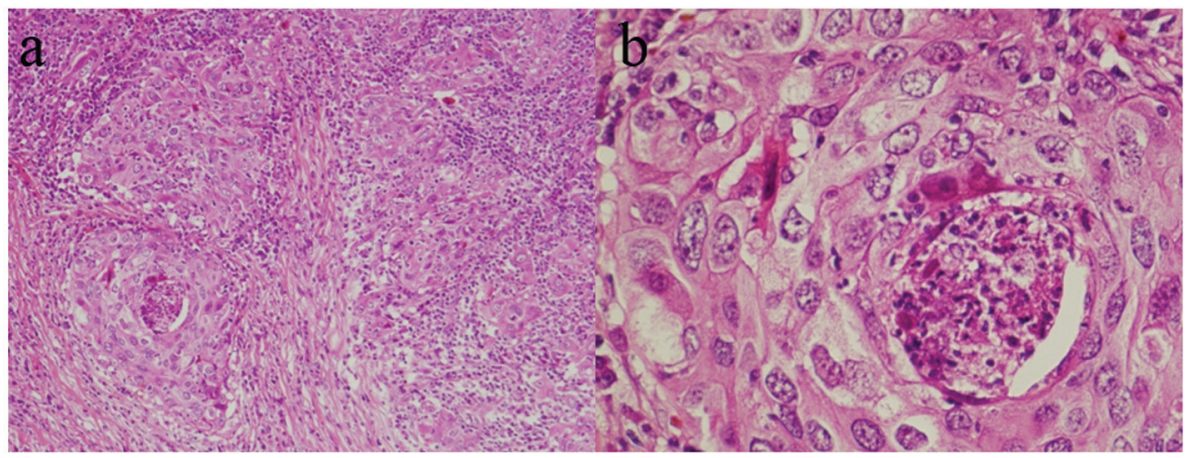

Figure 3. The squamous carcinoma showed solid nests of polygonal cells with keratinization (a) and consisted of large irregular nuclei with prominent nucleoli (b).

performed until May 2009 with 60 Gy divided into 30 fractions. CT in October 2009 demonstrated relapse of the thymic carcinoma without relapse of the $\mathrm{HCC}$ at the adrenal gland and the lymph nodes of the upper mediastinum and para-abdominal aorta. Systemic chemotherapy with the ADOC regimen, comprising cisplatin $50 \mathrm{mg} / \mathrm{m}^{2}$ and adriamycin $40 \mathrm{mg} / \mathrm{m}^{2}$ on day 1 , vincristine $0.6 \mathrm{mg} / \mathrm{m}^{2}$ on day 3 , and cyclophosphamide $700 \mathrm{mg} / \mathrm{m}^{2}$ on day 4 , was administrated twice until November 2009. However, the tumor progressed rapidly at each metastatic site, and the patient died of the pleuritis carcinomatosa in January 2010. Autopsy was not performed.

\section{Discussion}

We have described a case of thymic carcinoma located in the left lower anterior mediastinum, i.e. at the left cardiophrenic angle. Thymic carcinoma usually arises from the upper anterior mediastinum. The unusual location and the coexisting $\mathrm{HCC}$ in this case led to a delay in management of the mediastinal nodule. The incidence of solitary mediastinal metastasis from HCC without pulmonary involvement is relatively rare, accounting for only $2.6 \%$ of autopsy cases in Japan [11]. The frequent sites of metastasis from advanced HCC include the lung, locoregional lymph nodes, and bone. However, it has also been reported that the most common site of distant metastatic lymphadenopathy from HCC is the mediastinum [12].
The mediastinal nodule in the present patient was diagnosed preoperatively as lymph node metastasis from the HCC. An early interventional approach for the mediastinal nodule would have been possible if HCC had been absent. A thymic epithelial tumor can be considered as part of the differential diagnosis if a mediastinal nodule is first detected in the upper anterior mediastinum. We were able to select thymothymectomy rather than lymphadenectomy when the thymic epithelial tumor was suspected preoperatively. More intensive observation and early resection of the mediastinal nodule for diagnosis or cure would have been advisable even during treatment of the HCC.

The 7-month tumor-free interval between resection of the thymic carcinoma and detection of postoperative recurrence was very short in comparison with the 7-year interval between the initial detection and resection of the mediastinal nodule. The sudden and aggressive behavior of this tumor after resection suggests an alteration in the malignant character of the previously benign thymoma [13].

\section{Conclusion}

We have described an ectopic thymic carcinoma with coexisting HCC for which management was difficult. The unusual location of the thymic carcinoma and the synchronous malignancy led to a delay in surgical resection. Early intervention for any mediastinal nodule appearing during the course of
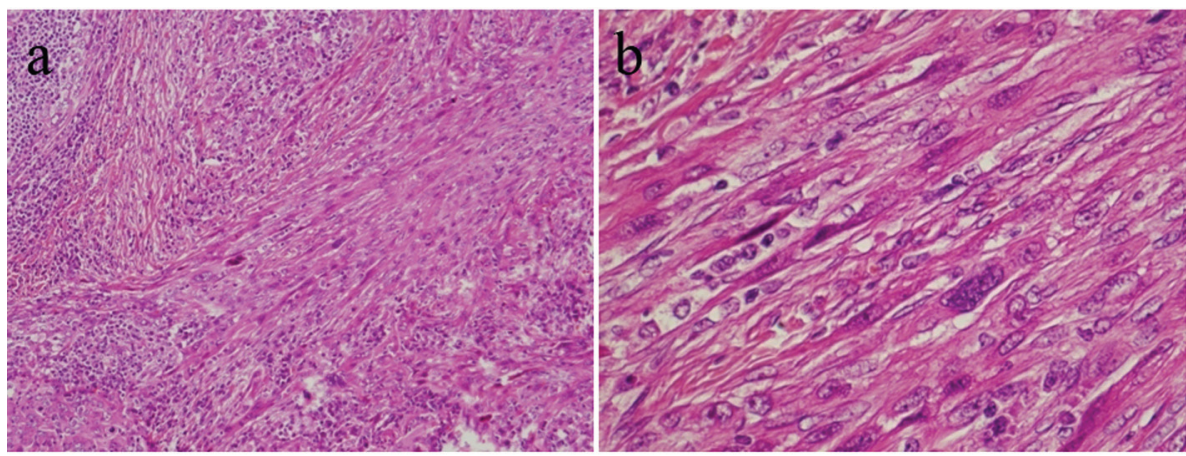

Figure 4. The sarcomatoid carcinoma showed palisade formation and polygonal spindle cells invading the surrounding area (a), and consisted of multinucleated cells (b). 
treatment for another tumor appears to be mandatory.

\section{Conflict of Interest}

Yasumichi Yamamoto and the other co-authors have no conflicts of interest to declare.

\section{Funding}

None.

\section{References}

1. HK Muller-Hermelink, P Engel, TT Kuo, Ph Strobel, A Marx, NL Harris, P Moller, et al. Tumours of the Thymus: Introduction. In: World Health Organization Classification of Tumours Pathology and Genetics of Tumours of the Lung, Pleura, Thymus and Heart. Edited by William D Travis, Elisabeth Brambilla, H Konrad, MullerHermelink, Curtis C Harris. Lyon: IARC Press; 2004.

2. Michal M, Neubauer L, Fakan F. Carcinoma arising in ectopic hamartomatous thymoma. An ultrastructural study. Pathol Res Pract. 1996;192(6):610-618; discussion 619621.

3. Hsu IL, Wu MH, Lai WW, Lin MY, Chang JM, Yen YT, Tseng YL. Cervical ectopic thymoma. J Thorac Cardiovasc Surg. 2007;133(6):1658-1659.

4. Calderon AM, Merchan JA, Rozo JC, Guerrero CI, Treistman B, Sulak LE, Cheong BY, et al. Intrapericardial primary thymic carcinoma in a 73-year-old man. Tex Heart Inst J. 2008;35(4):458-461.

5. Okuda M, Huang CL, Haba R, Yokomise H. Clear cell carcinoma originating from ectopic thymus. Gen Thorac
Cardiovasc Surg. 2009;57(5):269-271.

6. Yao WT, Chen CH, Lee JJ, Chen BF, Liu TP. Ectopic thymic carcinoma in the neck. Ann Thorac Surg. 2010;90(2):666-668.

7. Cappelli C, Tironi A, Marchetti GP, Pirola I, De Martino E, Delbarba A, Castellano M, et al. Aggressive thyroid carcinoma showing thymic-like differentiation (CASTLE): case report and review of the literature. Endocr J. 2008;55(4):685-690.

8. Yerly S, Lobrinus JA, Bongiovanni M, Becker M, Zare M, Granger P, Pusztaszeri M. A carcinoma showing thymus-like elements of the thyroid arising in close association with solid cell nests: evidence for a precursor lesion? Thyroid. 2013;23(4):511-516.

9. Fernandez-Ruiz M, Guerra-Vales JM, Castelbon-Fernandez FJ, Llenas-Garcia J, Caurcel-Diaz L, ColinaRuizdelgado F. Multiple primary malignancies in Spanish patients with hepatocellular carcinoma: analysis of a hospital-based tumor registry. J Gastroenterol Hepatol. 2009;24(8):1424-1430.

10. Takayasu K, Kasugai H, Ikeya S, Muramatsu Y, Moriyama N, Makuuchi M, Yamazaki S, et al. A clinical and radiologic study of primary liver cancer associated with extrahepatic primary cancer. Cancer. 1992;69(1):45-51.

11. Takada K, Horita S, Meguro T, Inoue Y, Gouda T, Nakamura H, Maruya M, et al. [An autopsy case of hepatocellular carcinoma with a solitary mediastinal lymph node metastasis]. Nihon Shokakibyo Gakkai Zasshi. 2003;100(2):177-184.

12. Katyal S, Oliver JH, 3rd, Peterson MS, Ferris JV, Carr BS, Baron RL. Extrahepatic metastases of hepatocellular carcinoma. Radiology. 2000;216(3):698-703.

13. Suster S, Moran CA. Primary thymic epithelial neoplasms showing combined features of thymoma and thymic carcinoma. A clinicopathologic study of 22 cases. Am J Surg Pathol. 1996;20(12):1469-1480. 